\title{
A 300 Mrad total-ionizing dose experiment for lasers used for holographic memories
}

\author{
Tomoya Akabe and Minoru Watanabe \\ Electrical and Electronic Engineering \\ Shizuoka University \\ 3-5-1 Johoku, Hamamatsu, Shizuoka 432-8561, Japan
}

\begin{abstract}
A Abstract - Recently, optically reconfigurable gate arrays (ORGAs) have been under development to produce radiationhardened field programmable gate arrays (FPGAs). An ORGA consists of a laser array, a holographic memory, and a programmable gate array VLSI. Since holographic memories are very robust against radiation compared with semiconductor devices, the ORGA exploits the radiation tolerance of holographic memories to increase the radiation tolerance of the entire ORGA device. This paper presents experimentally obtained results of a part of an ORGA device. We have conducted an experiment using a cobalt 60 gamma radiation source to confirm the total ionizing dose (TID) tolerance of an ORGA. Results show that a laser used in the ORGA can function correctly even after receiving a $300 \mathrm{Mrad}$ TID.
\end{abstract}

Keywords-Radiation hardened field programmable gate arrays, Optically reconfigurable gate arrays

\section{Introduction}

Fukushima Daiichi Nuclear Power Plant has presented an accident by which the nuclear fuel assembly has melted [1][2]. Currently, highly radiation-tolerant embedded systems are required for robots working at the nuclear power plant to improve the situation and to decommission the Fukushima Daiichi Nuclear Power Plant.

Recently, radiation-hardened field programmable gate arrays (FPGAs) having up to $1 \mathrm{Mrad}$ total ionizing dose (TID) tolerance are available [3]-[7]. However, since the radiation levels of some areas close to containment vessels in the Fukushima Daiichi Nuclear Power Plant are presumed to have radiation levels higher than $100 \mathrm{~Sv} / \mathrm{h}$, an embedded system constructed for space-grade devices would not have sufficient radiation tolerance for use in such areas.

Therefore, heavily shielded materials are needed for use with the embedded devices. Semiconductor devices would otherwise fail quickly in a $100 \mathrm{~Sv} / \mathrm{h}$ environment. This represents an important current issue for work in this field. To increase the radiation tolerance of semiconductor devices, we have been developing an optically reconfigurable gate array (ORGA) having field programmable gate array (FPGA) function [8][9].

An ORGA consists of a laser array, a holographic memory, and a programmable gate array VLSI. Since holographic memories are much more robust against radiation than semiconductor devices, the ORGA exploits radiation tolerances of the holographic memories to increase the radiation tolerance of the entire ORGA device.

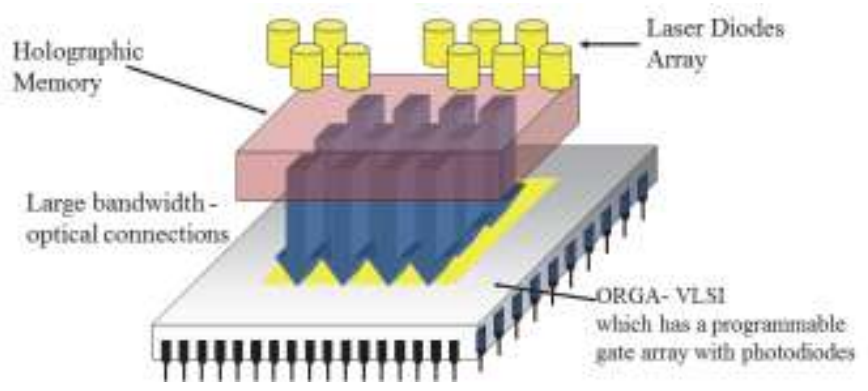

Fig.1 Overview of an optically reconfigurable gate array (ORGA).

TABLE I

Specifications of an optically reconfigurable gate array VLSI.

\begin{tabular}{ll}
\hline Technology & $\begin{array}{l}0.35 \mu \mathrm{m} \text { double-poly } \\
\text { triple-metal CMOS process }\end{array}$ \\
\hline Chip size & $4.9 \mathrm{~mm} \times 4.9 \mathrm{~mm}$ \\
\hline Photodiode size & $25.5 \mu \mathrm{m} \times 25.5 \mu \mathrm{m}$ \\
\hline Distance between photodiodes & $90 \mu \mathrm{m}$ \\
\hline $\begin{array}{l}\text { Number of photodiodes } \\
\text { Implementation Area of } \\
\text { Photodiodes }\end{array}$ & 340 \\
\hline $\begin{array}{l}\text { Implementation Area of } \\
\text { Photodiode Cells }\end{array}$ & $221.085 \mu^{2}, 1.8 \%$ \\
\hline $\begin{array}{l}\text { Implementation Area of } \\
\text { Reconfiguration Circuits }\end{array}$ & $659,175 \mu \mathrm{m}^{2}, 5.3 \%$ \\
\hline
\end{tabular}

Experimentally obtained results obtained after irradiation of ORGA device components confirm the TID tolerance of an ORGA using cobalt 60 gamma radiation source. A laser used in the ORGA can function correctly after sustaining a $300 \mathrm{Mrad}$ TID.

\section{Optically reconfigurable gate array}

Figure 1 presents an overview of an ORGA. It is a type of multi-context field programmable gate array (FPGA). 


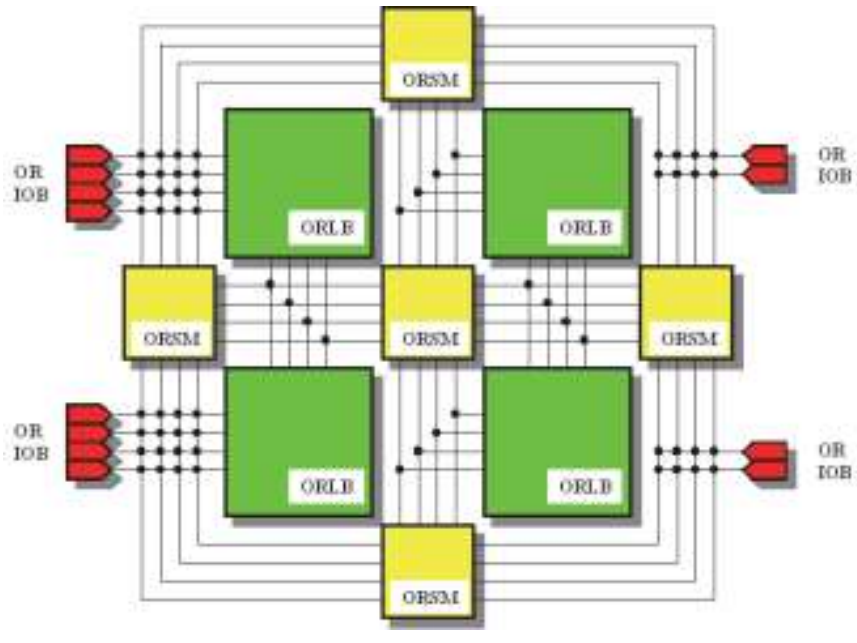

(a)

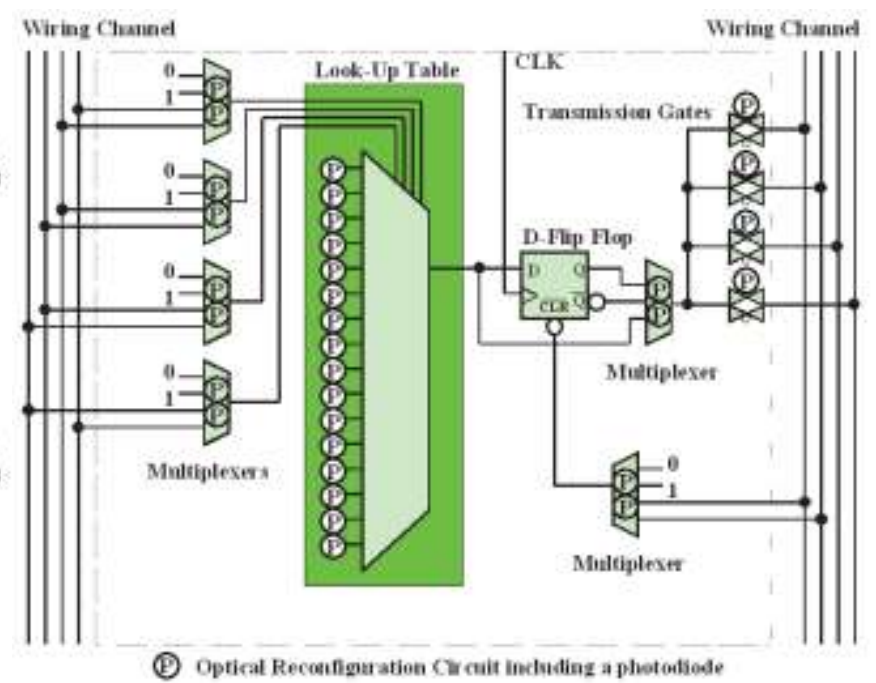

(b)

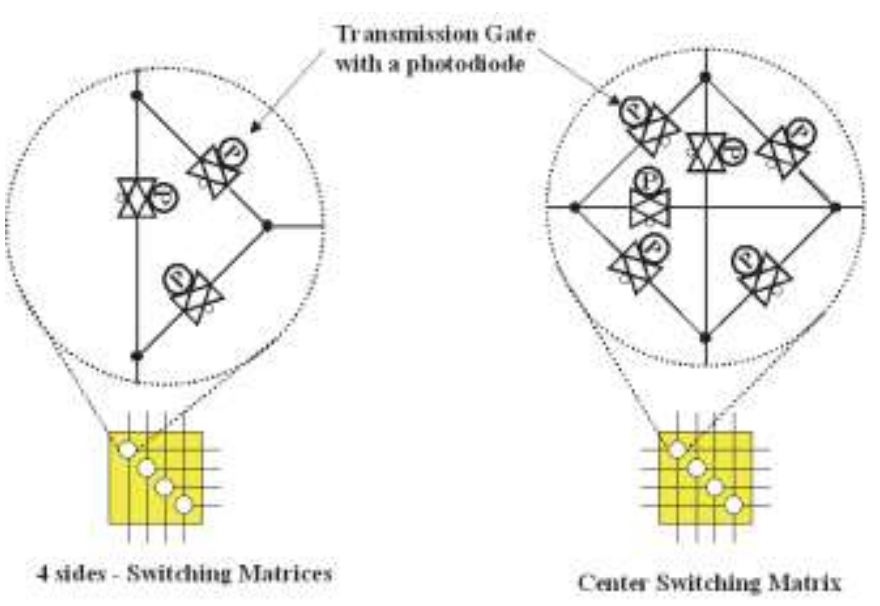

(c)

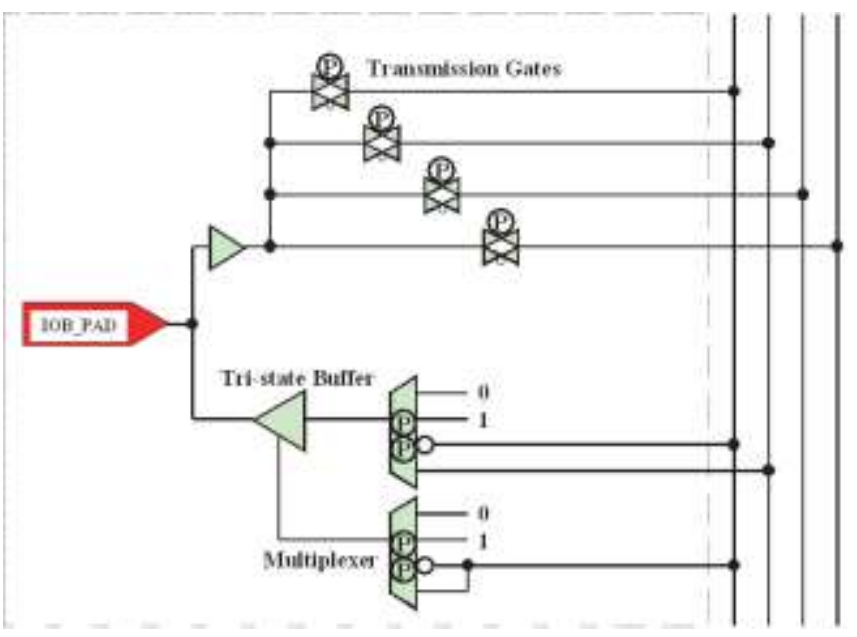

(d)

Fig. 2 Gate-array structure of an ORGA. Panels (a), (b), (c), and (d) respectively depict block diagrams of a gate array, an optically reconfigurable logic block, an optically reconfigurable switching matrix, and an optically reconfigurable I/O bit.

Actually, ORGAs are based on an architecture that includes look-up-tables and switching matrices. Therefore, the basic function of the ORGA is identical to that of FPGAs. Sicne the ORGA has look-up-tables and flip-flops just as FPGAs do, any circuit can be implemented onto an ORGA. However, the ORGA can be optically reconfigured. The ORGA architecture uses an optical holographic memory. Since many configuration contexts can be stored on a holographic memory, a larger virtual circuit over the size of the ORGA-VLSI can be implemented. Therefore, the ORGA performance is higher than that of FPGAs. Moreover, the holographic memory has high radiation tolerance. By exploiting the high radiation tolerance of a holographic memory, the entire radiation tolerance of the ORGA can be increased drastically. Since the holographic memories are distributed memory, each bit of information is generated from almost the entire region of a holographic memory. Every bright bit or binary state high can be generated with numerous same-phase waves, whereas every dark bit can be generated with waves of various phases. Since the reading operation can be considered as a majority-voting operation, information can be read from holographic memories very robustly. Therefore, even if half of a holographic memory is removed after something is recorded on it, the complete and correct information can be read out from the remaining half of the holographic memory. Therefore, even if ORGAs are exposed to extreme radiation, the correct configuration can be achieved using a robust holographic memory.

In the ORGA, the reconfiguration contexts are addressed by a laser array mounted on the top of the holographic memory. The diffraction pattern from the holographic memory can be received as a reconfiguration context on a photodiode-array implemented on a programmable gate array VLSI. This architecture allows nanosecond-order reconfiguration and multiple reconfiguration contexts. The ORGA-VLSI chip was fabricated using a $0.35 \mu \mathrm{m}$ triplemetal CMOS process. Its specifications are presented in 


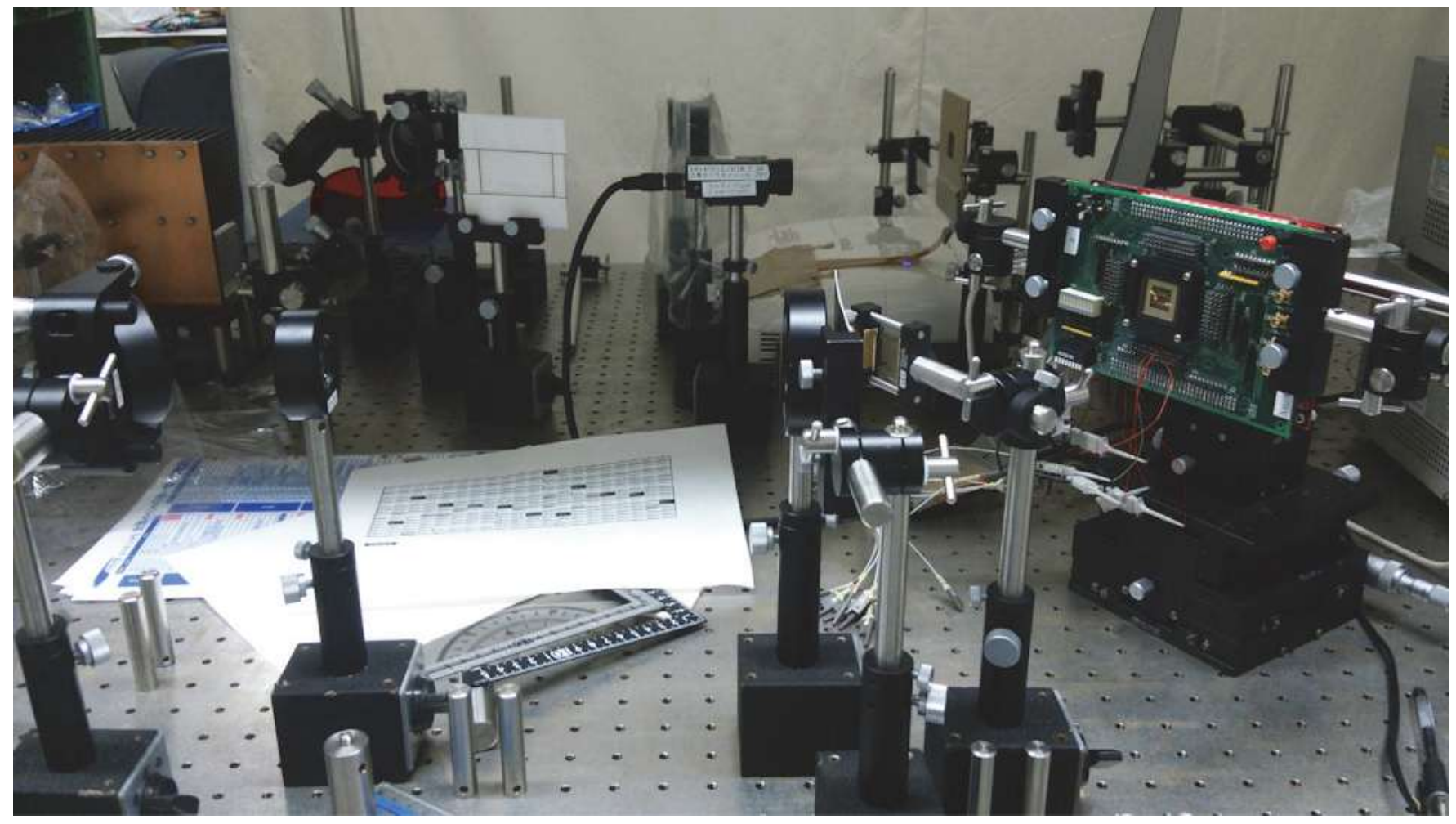

Fig. 3 Configuration experiment for an optically reconfigurable gate array.
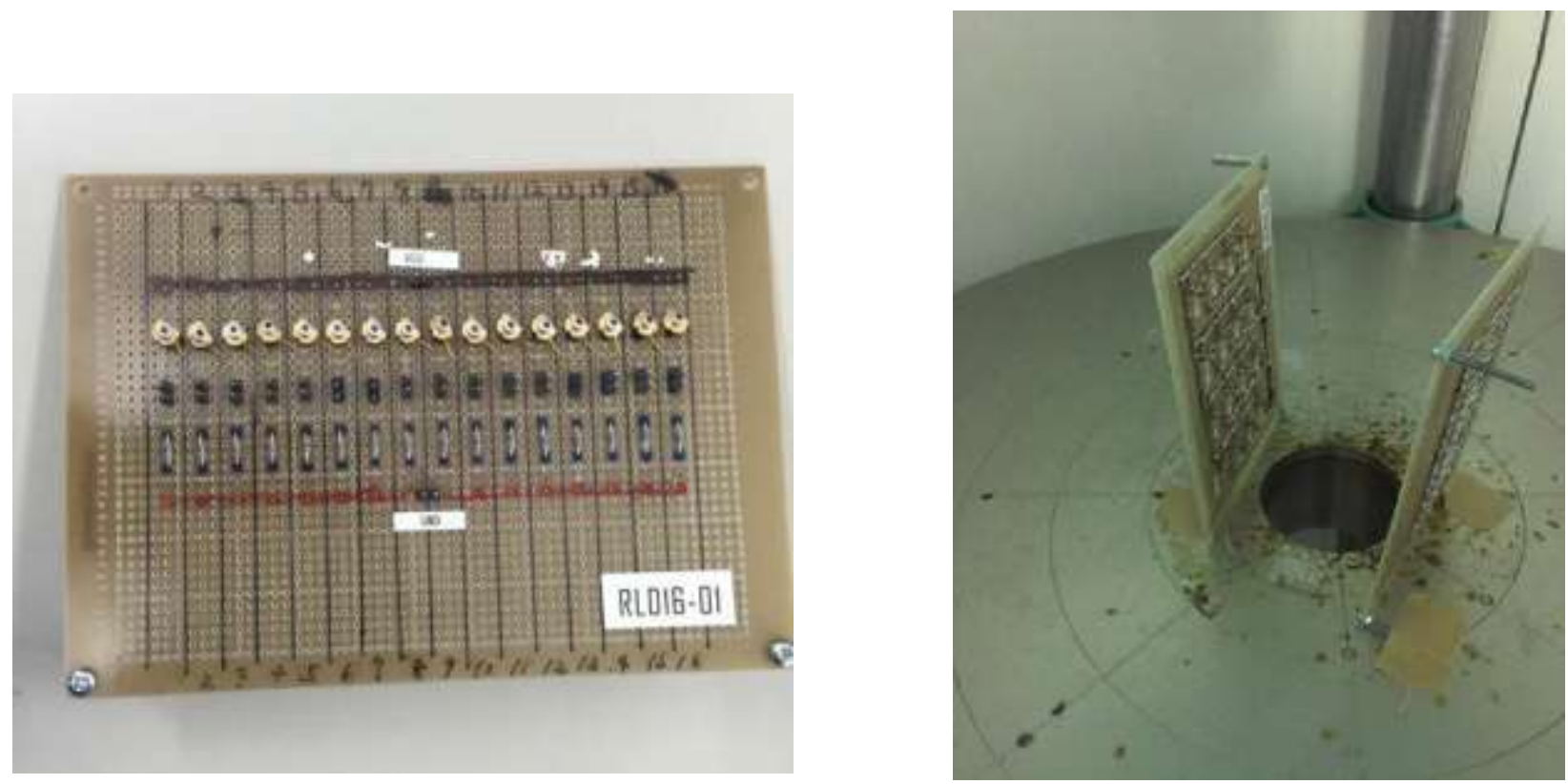

Fig. 4 Photograph of a laser array with 16 lasers.

Fig. 5 Photograph of a laser array in a Co60 radiation environment.

Table 1. Figure 2 depicts the gate array structure of the fabricated ORGA-VLSI chip, which consists of four logic blocks, five switching matrices, and $12 \mathrm{I} / \mathrm{O}$ bits, as portrayed in Fig. 2(a). The VLSI chip functionality is fundamentally identical to that of typical FPGAs. However, each programming element of all blocks of the ORGA-VLSI is connected to a photodiode. All related block diagrams are depicted in Figs. 2(b)-2(d). A logic block comprises a fourinput-one-output look-up table (LUT), four multiplexers, and a delay flip-flop with a reset function. These functions are optically reconfigurable by 40 optical reconfiguration circuits. Similarly, switching matrices can be reconfigured optically. One four-directional and four three-directional switching matrices were implemented in the gate array. The four-direction and three-direction switching matrices have, respectively, 24 and 12 optical connections. All programming elements can be optically reconfigured simultaneously. Each I/O block is also controlled by nine optical connections. For this fabrication, we designed the distance between each photodiode as $90 \mu \mathrm{m}$; the photodiode 
is $25.5 \mu \mathrm{m} \times 25.5 \mu \mathrm{m}$. The total number of photodiodes is 340. The gate array's gate count is 68 .

\section{Total-ionizing-dose tolerance of a laser array}

A laser array in an ORGA is used for addressing configuration contexts inside a holographic memory. As explained earlier, holographic memory is very robust against radiation. To date, we have confirmed 200 Mrad TID tolerance. For the experiment described herein, we developed a laser array including 16 lasers (DL-3247-165: Tottori Sanyo Electric Co., Ltd.) applied for such radiationhardened holographic memories on ORGAs, as shown in Fig. 4. The laser wavelength and laser power are $650 \mathrm{~nm}$ and $7 \mathrm{~mW}$, respectively. The laser array was set at $0.05 \mathrm{~m}$ from a cobalt 60 gamma radiation source and was exposed to gamma radiation, as presented in Fig. 5. Then the VI characteristics and light power of each laser were measured. The laser power never decreased. It continued to operate at 7 $\mathrm{mW}$ up to $300 \mathrm{Mrad}$.

Results show that the laser can continue to function correctly up to $300 \mathrm{Mrad}$ TID. Although the optical powers of four lasers among 16 lasers were reduced by exposure to over 300 Mrad gamma radiation, a few spare lasers can rectify that shortcoming: the power of the other lasers was maintained as equal to the initial level up to $300 \mathrm{Mrad}$.

\section{Conclusion}

Recently, ORGAs have been under development to realize radiation-hardened field programmable gate arrays (FPGAs) An ORGA consists of a laser array, a holographic memory, and a programmable gate array VLSI. Since holographic memories are much more robust against radiation than semiconductor devices, an ORGA can exploit the radiation tolerances of the holographic memories and thereby increase the radiation tolerance of entire of the ORGA device. This report describes experimentally obtained results for an ORGA device. We conducted an experiment to confirm the TID tolerance of an ORGA using a Cobalt 60 gamma radiation source. Results show that a laser used in the ORGA can function correctly even after it has received 300 Mrad TID. The radiation tolerance is 300 times higher than that of currently available radiation-hardened devices.

\section{Acknowledgments}

This research was partly supported by the Ministry of Education, Science, Sports and Culture, Grant-in-Aid for Scientific Research (B), No. 15H02676. The VLSI chip in this study was fabricated in the chip fabrication program of VLSI Design and Education Center (VDEC), the University of Tokyo in collaboration with Rohm Co. Ltd. and Toppan Printing Co. Ltd.

\section{References}

[1] Robert C. Baumann, "Determining the Impact of Alpha-ParticleEmitting Contamination From the Fukushima-Daiichi Disaster on Japanese Semiconductor Manufacturing Sites," IEEE Transactions on Nuclear Science, Vol. 59, Issue 4, pp. 1186 - 1196, 2012.

[2] Robert C. Baumann, "Determining the impact of alpha-particleemitting contamination from the Fukushima Daiichi disaster on Japanese manufacturing sites," European Conference on Radiation and Its Effects on Components and Systems, pp. 784 - 787, 2011.

[3] P. Calvel, P. Lamothe, C. Barillot, R. Ecoffet, S. Duzellier, E. G. Stassinopoulos, "Space radiation evaluation of 16 Mbit DRAMs for mass memory applications," IEEE Transactions on Nuclear Science, Vol. 41, Issue 6, pp. 2267-2271, 1994.

[4] J. R. Schwank, M. R. Shaneyfelt, J. A. Felix, P. E. Dodd, J. Baggio, V. Ferlet-Cavrois, P. Paillet, G. L. Hash, R. S. Flores, L. W. Massengill, E. Blackmore, "Effects of Total Dose Irradiation on Single-Event Upset Hardness," IEEE Transactions on Nuclear Science, Volume: 53, Issue 4, pp. 1772-1778, 2006.

[5] D.A. Adams, J.T. Smith, J.R. Murray, M.H. White, S. Wrazien, "Design, fabrication and characterization of high density radiation hardened SONOS/CMOS EEPROMs for space and military systems," Non-Volatile Memory Technology Symposium, pp. 60-65, 2004.

[6] D. Wellekens, G. Groeseneken, J. Van Houdt, H. E. Maes, "Single poly cell as the best choice for radiation-hard floating gate EEPROM technology," IEEE Transactions on Nuclear Science, Vol. 40, Issue 6, pp. 1619-1627, 1993.

[7] Northrop Grumman Corp., "Radiation Hardened 128K x 8 CMOS EEPROM," http://www.northropgrumman.com/Capabilities/Radiation HardenedEEPROMS/Documents/ 1mbiteeprom.pdf.

[8] M. Watanabe, "Quality recovery method of interference patterns generated from faulty MEMS spatial light modulators," IEEE/OSA Journal of Lightwave Technology, vol. 34, Issue 3, pp. 910-917, 2016.

[9] A. Ogiwara, M. Watanabe, "Formation of holographic polymerdispersed liquid crystal memory by angle-multiplexing recording for optically reconfigurable gate arrays," Applied Optics, Vol. 54, Issue 36, pp. 10623-10629, 2015. 